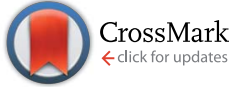

Cite this: RSC Adv., 2017, 7, 15527

Received 5th January 2017

Accepted 27th February 2017

DOI: 10.1039/c7ra00146k

rsc.li/rsc-advances

\section{Numerical investigation on the elastic-plastic transition of a cylindrical coating/substrate composite under normal compression}

\begin{abstract}
Yongtao Mu, Ming Liu* and Yongqiang Zhao
In order to study the yielding resistance of a coated component in line contact, a cylindrical coating/substrate system contacting a rigid plane was simulated via a plane-strain model. For super-thick coatings, the critical load, contact width and interference were derived for the onset of plastic yielding on the axis of symmetry by a function approximation method. The results indicated the geometrical and mechanical properties of the loaded coating displayed an important influence on these threshold values. For thinner coatings, the Von Mises stress distribution along the axis of symmetry was investigated using a finite element method, of which a pronounced discontinuity at the interface was found by increasing the elasticity modulus of the coating relative to the substrate. Furthermore, an additional stress was induced by the binding constraints imposed at the coating/substrate interface, thus resulting in a weakened resistance to the plastic initiation in relatively thin coatings. In light of finite element results, the initial yield position may be located either within the coating, in the substrate or at the interface between them with the ratio $t / R$ of coating thickness over the substrate radius changing. Besides, when $t / R$ was very small, the total interference of the coated cylinder was mainly attributed to the substrate deformation. With $t / R$ ratio becoming larger, the coating compression became comparable to the substrate value. When $t / R$ had large values, the coating contraction served as a dominant contributor to the composite interference.
\end{abstract}

\section{Introduction}

Thin film coatings are widely used in many engineering applications to enhance the tribological performance of metal parts in contact and in relative sliding. ${ }^{1-8}$ The elastic-plastic deformation of a coating/substrate system is a major factor that affects the tribology properties of a contact surface. The elastic-plastic transition may occur either within the coating, in the substrate or at their interface, accordingly it can lead to the fracture of the coating, or deformation of the substrate, or even coating delamination from the substrate. In many cases, the mechanical components interact with counterparts that are in line contact during a working process, such as cylindrical gears, ${ }^{9}$ bearings ${ }^{10}$ and clutches, ${ }^{11}$ etc., which have a complex stress and strain mechanism under normal loading. Therefore, it is imperative to study the plasticity inception of coating/substrate composites that are in line contact for the failure analysis of loaded elements.

For line contact problems, a cylinder pressing a plane is commonly adopted as the basic model for researching the deformation mechanism of longitudinal objects, as shown in Fig. 1. Many mechanical investigations have been accomplished with regard to the line contact of uniform materials through the general model stated above. Liu et al. studied the adhesive contact

School of Mechatronics Engineering, Harbin Institute of Technology, Harbin 150001, People's Republic of China. E-mail: liuming@hit.edu.cn; Tel: +8645186403799 mechanics of a long rigid cylinder on an infinite half space comprising incompressible elastic material based on small strain theory. ${ }^{12}$ Saito et al. investigated the critical conditions of rolling resistance in respect to an elastic micro-cylinder in adhesional contact with a rigid surface using a combined theoretical/ computational approach. ${ }^{13}$ Jin et al. analytically studied the adhesive contact of a rigid cylinder on an elastic power-law graded half-space with the theory of a weakly singular integral equation and an orthogonal polynomial method. ${ }^{14}$ Zhupanska et al. presented an exact solution to the problem of indentation with friction of a rigid cylinder with an elastic half-space. ${ }^{15}$ Qiu et al. investigated the skew sliding of an elastic cylinder along an elastically similar half-plane and found the effect of surface strain on

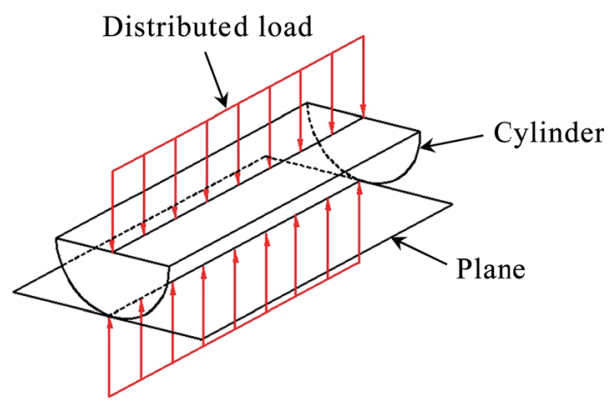

Fig. 1 Line contact model of a cylinder pressing a plane. 
the local slip angle. ${ }^{16} \mathrm{Li}$ et al. built a finite element model for the spatial contact behavior of a plate and a cylinder on account of gear transmission, which illustrated the influence of element size on contact force in different curvatures. ${ }^{17}$ Pereira et al. proposed an analytical model describing the cylindrical contact force as an explicit function of the penetration. ${ }^{18}$ Chiu et al. presented a solution for the three-dimensional cylindrical contact by a numerical iterative technique, which determined the contact pressure, as well as an elastic approach in considering the cylinder effect. ${ }^{19}$ Hunter studied the rolling contact of a rigid cylinder with a half space, considering the asymmetry through introducing viscoelastic effects. ${ }^{20}$ Doca et al. studied the frictional contact problems between a mild steel cylinder and an aluminium alloy block undergoing finite elastoplastic strains, ${ }^{21}$ and compared the predictions with experimental observations. ${ }^{22}$ Chen et al. found a possible rolling of an elastic cylinder adhering on an elastic substrate subject to a strain gradient and established a criterion for the initiation of rolling. ${ }^{23}$

With regards to the involved materials having a nonhomogeneous characteristic, Meijers presented an asymptotic solution for the contact problem between a rigid cylinder and an elastic layer fastened to a rigid base, by assuming no friction and a long enough dimension to ensure a plane strain. ${ }^{24}$ Stupkiewicz et al. employed a plane-strain element to simulate the contact problem of a rigid cylinder sliding against a coated layer, where the elastohydrodynamic lubrication effect was taken into account. ${ }^{25}$ Proudhon et al. simulated the wear of a coated plate in contact with an elastic cylinder by a plain strain finite element method using an updated Lagrangian formulation. ${ }^{26}$ Saka et al. studied the contact behavior of a rigid cylinder indenting an elastoplastic two-layer half-space by a finite element method. ${ }^{27,28}$ Gupta et al. calculated the numerical solutions of the contact pressure and contact area by using an integral transform technique for the problem of a coated flat pressed by an elastic cylinder. ${ }^{29}$ Alinia et al. investigated the subsurface stress field in a functionally graded coating-substrate system induced by a rolling rigid cylinder with a Fourier transform technique, where the effects of the stiffness ratio and the interface shear modulus ratio of the coating and substrate on the stress distributions were analyzed throughout the system. ${ }^{30}$ Guler et al. investigated the frictional contact problem of a graded coating/substrate system under the action of a sliding cylinder, ${ }^{31}$ furthermore, the rolling contact problems were considered for all possible stick/slip regimes by comprehensive parametric analysis. ${ }^{32,33}$ Cole et al. studied the contact pressure distribution between a smooth cylinder and a coated flat, and they also found a numerical solution to the elastic stress field in a multi-layer system. ${ }^{\mathbf{3 4}}$ Komvopoulos carried out an elastic-plastic finite element analysis on a TiN/Ti system indented by a rigid cylinder, finding that the coating thickness had a significant effect on the initiation and development of the plastic zone. ${ }^{35}$ Yang et al. performed a dynamic finite element analysis on an elastic-plastic multilayered system indented by a rigid cylinder via a plane-strain model. ${ }^{36}$ Gong et al. researched the surface cracking in a multilayered medium due to the sliding of a cylindrical rigid asperity using linear elastic fracture mechanics and a finite element method. ${ }^{37}$ Njiwa et al. modeled a coated flat under cylindrical indentation using a boundary element approach, demonstrating the sub-surface stress field and its discontinuity at the coatingsubstrate interface depended crucially on the relative Young's moduli, film thickness and Poisson ratio of the coating and substrate. ${ }^{38}$ Djabella et al. performed a finite element analysis for the elastic contact between a coating/substrate flat and a rigid cylindrical indenter based on plane strain model, where the frictional sliding at the coated surface was considered under a combined normal and tangential load..$^{39,40}$ Jahedi et al. carried out an elastic finite element investigation for a graded coated substrate in frictional contact with rigid cylindrical roller. ${ }^{41}$ Moreover, mechanical investigations about the line contact were studied by two coated cylinders in relative sliding or rolling. ${ }^{\mathbf{4 2 , 4 3}}$

As can be seen from the aforementioned literature review, for a coating/substrate composite, most of the contact investigations in cylinder on plane model were focused on a coated flat and in the elastic range, but relatively few calculations have solved this for a coated cylinder, especially with regards to the plastic yielding. Therefore, in the current research the plasticity inception of a cylindrical coating/substrate composite in contact with a rigid flat was simulated in light of a plane strain model, as well as changing the ratio of the coating thickness over the substrate radius and the relative mechanical properties of the composite system. Some critical parameters were obtained for the large value of the coating thickness relative to substrate radius. Nevertheless, when the coating was relatively thin, the elastic-plastic transition along the axis of symmetry of the loaded cylinder was studied by a finite element method, and the additional stress and its discontinuity at the coatingsubstrate interface were observed under various simulation parameters. The present achievements may open the way for wider research on the contact mechanics of coated cylindrical components, thus avoiding the need of an inaccurate trial and error approach for designing the optimal coating.

\section{Hertz contact model}

\subsection{Contact schematic}

For the super coating thickness relative to the substrate radius, the substrate deformation is negligible due to its far distance from the contact zone, so the contact model of a coated cylinder and a rigid flat can consider only the coating strain on basis of Hertz theory. Fig. 2 schematically presents a plane-strain model for the cylindrical coating loaded by a rigid flat under perfect slip conditions. The coating material is assumed to be elastic and isotropic before the onset of plastic yielding, of which the Poisson's ratio $\nu$ varies in the range of 0.2 to 0.5 . Nothing but normal load is applied to the rigid flat. It is adequate to take only half of the semicircle into account due to the axisymmetric cylinder and loading. The degrees of freedom of the semicircular base are restricted in vertical and radial directions. Meanwhile, the degrees of freedom of the axis of symmetry are restricted in the radial direction. The rest of the quarter circle is free except for a normal compression exerted by the contacting rigid flat. The dashed and solid lines in the schematic denote the outlines of the bodies in contact with each other before and after loading, respectively. The contact area with a width $2 b$ and an interference 


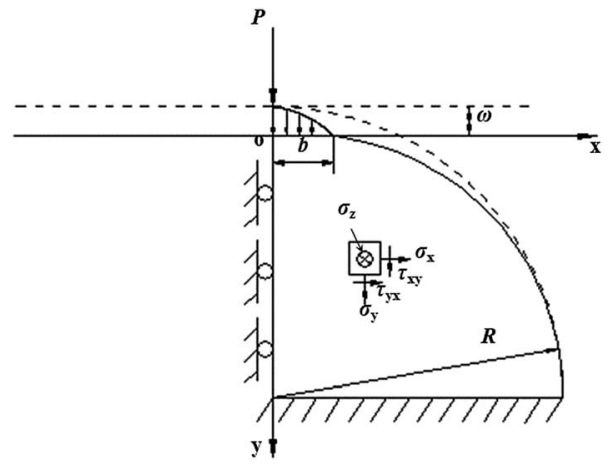

Fig. 2 Hertz contact between a cylindrical coating and a rigid plane.

$\omega$ correspond to the normal load $P$. Both $\omega$ and $b$ are supposed to be much smaller than the composite radius $R$. A Cartesian coordinate system is established on the quarter circle after loading, whose origin o is located at the center of the contact area and its $x$-axis, $y$-axis and $z$-axis are parallel to the radial, vertical and axial directions of the cylindrical plane, respectively.

\subsection{Stress distribution along the axis of symmetry}

According to the Hertz solution, ${ }^{44}$ the contact pressure of the compressed coating has a semi-elliptical distribution in a planestrain model, that is

$$
p(x)=\frac{p_{\mathrm{m}}\left(b^{2}-x^{2}\right)^{1 / 2}}{b},|x| \leq b
$$

where the variable $x$ represents a radial distance measured from the center of the contact area and the parameter $p_{\mathrm{m}}$ represents the maximum contact pressure at the contact center, which is given by

$$
p_{\mathrm{m}}=\frac{2 P}{\pi b}
$$

The half width $b$ of the contact area is written as

$$
b=2\left[\frac{P R\left(1-\nu^{2}\right)}{\pi E}\right]^{1 / 2}
$$

where $\nu$ and $E$ signify the Poisson's ratio and Young modulus of the coating material, respectively. A Goldsmith model is adopted to calculate the coating interference $\omega$, which is given by ref. 45

$$
\begin{aligned}
& \omega=\frac{P\left(1-\nu^{2}\right)}{\pi E}\left\{\ln \left[\frac{\pi E}{P R\left(1-\nu^{2}\right)}\right]+1\right\} \quad \text { (4) } \begin{array}{l}
\text { obtained as } \\
\text { obs }
\end{array} \\
& \left.\qquad \begin{array}{l}
\frac{\sigma_{\mathrm{eq}}}{p_{\mathrm{m}}}=\frac{\sqrt{2}}{2}\left(2(y / b)-\frac{2(y / b)^{2}}{\left((y / b)^{2}+1\right)^{1 / 2}}\right)^{2}+\left(2(\nu-1)(y / b)+\frac{1-2 \nu+(2-2 \nu)(y / b)^{2}}{\left((y / b)^{2}+1\right)^{2}}\right)^{1 / 2} \\
+\left(2 \nu(y / b)+\frac{1-2 \nu-2 \nu(y / b)^{2}}{\left((y / b)^{2}+1\right)^{1 / 2}}\right)^{2}
\end{array}\right)
\end{aligned}
$$

Since the axial deformation is restricted for the plane-strain model, the shear stress components $\tau_{z x}, \tau_{z y}, \tau_{x z}, \tau_{y z}$ and the axial strain $\varepsilon_{z}$ are equal to zero, that is

$$
\left\{\begin{array}{l}
\tau_{z x}=\tau_{z y}=\tau_{x z}=\tau_{y z}=0 \\
\varepsilon_{z}=0
\end{array}\right.
$$

So, based on Hooke's law, the normal stress components $\sigma_{x}$, $\sigma_{y}, \sigma_{z}$ in the plane-strain model satisfy a relation of

$$
\sigma_{z}=\nu\left(\sigma_{x}+\sigma_{y}\right)
$$

Because the shear stress components $\tau_{x y}$ and $\tau_{y x}$ on the axis of symmetry of the coating are all equal to zero, ${ }^{\mathbf{4 4}}$ combining eqn (5), the normal stress components $\sigma_{x}, \sigma_{y}, \sigma_{z}$ on the axis of symmetry are the three principal stresses, and the directions of the coordinate axis are the principal directions. Then the stress field along the $y$-axis which is normal to the contact area at the contact center is written as ref. 44

$$
\left\{\begin{array}{l}
\sigma_{x}=-\frac{p_{\mathrm{m}}}{b}\left[\frac{b^{2}+2 y^{2}}{\left(y^{2}+b^{2}\right)^{1 / 2}}-2 y\right] \\
\sigma_{y}=-\frac{b p_{\mathrm{m}}}{\left(y^{2}+b^{2}\right)^{1 / 2}} \\
\sigma_{z}=\nu\left(\sigma_{x}+\sigma_{y}\right) \\
\tau_{x y}=\tau_{y z}=\tau_{z x}=0
\end{array}\right.
$$

where variable $y$ represents a vertical distance measured from the contact centre.

\subsection{Yielding inception and critical parameters}

The Von Mises criterion is proposed to evaluate the elasticplastic transition of the coating materials, ${ }^{\mathbf{4 6}}$ which is expressed as

$$
Y=\sigma_{\mathrm{eq}}=\left\{\frac{1}{2}\left[\left(\sigma_{1}-\sigma_{2}\right)^{2}+\left(\sigma_{2}-\sigma_{3}\right)^{2}+\left(\sigma_{1}-\sigma_{3}\right)^{2}\right]\right\}^{1 / 2}
$$

where $\sigma_{\text {eq }}$ is the equivalent Von Mises stress and $Y$ is the yield strength of the coating material, $\sigma_{1}, \sigma_{2}$ and $\sigma_{3}$ represent the principal stresses. Accordingly, for the stress field given in eqn (7), by substituting the principal stresses $\sigma_{x}, \sigma_{y}$ and $\sigma_{z}$ into eqn (8), a dimensionless stress $\sigma_{\mathrm{eq}} / p_{\mathrm{m}}$ as a function of the dimensionless distance $y / b$ for a given value of variable $\nu$ is 


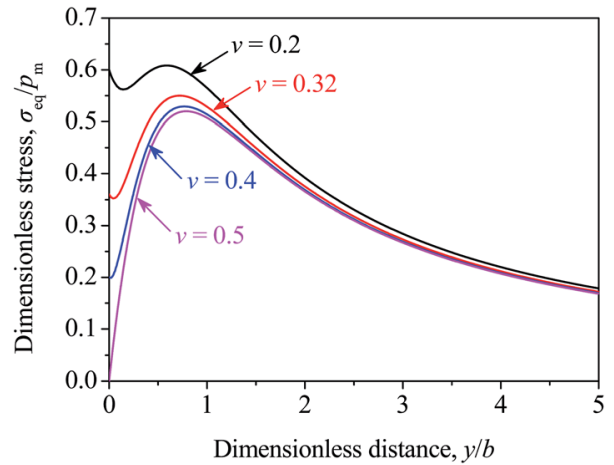

Fig. 3 Dimensionless stress $\sigma_{\mathrm{eq}} / p_{\mathrm{m}}$ vs. the dimensionless distance $y /$ $b$ for various values of Poisson's ratio $\nu$.

Fig. 3 presents the function graph of the dimensionless stress $\sigma_{\mathrm{eq}} / p_{\mathrm{m}} v s$. the dimensionless distance $y / b$ for various values of Poisson's ratio $\nu$. Apparently, for the values of the Poisson's ratio of $0.2,0.32,0.4$ and 0.5 , the dimensionless stresses first decrease slightly from the contact center (the greater the value of the Poisson's ratio, the smaller the decrease; for the Poisson's ratio of 0.5 , the decrease is zero due to incompressibility of the material), then increase until their maximum values and afterwards approach zero along the $y$-axis. Moreover, the maximum value $\left(\sigma_{\mathrm{eq}} / p_{\mathrm{m}}\right)_{\max }$ decreases with the increasing Poisson's ratio, however the corresponding dimensionless location $y / b=(y / b)_{\mathrm{p}}$ shows a growth tendency.

Because the equivalent Von Mises stress $\sigma_{\text {eq }}$ is less than or equal to the yield strength $Y$ of the coating material before the plasticity inception, the peak value $\left(\sigma_{\mathrm{eq}} / p_{\mathrm{m}}\right)_{\max }$ is equal to the value of $Y / p_{\mathrm{m}}$ at the elastic terminus for the different values of Poisson's ratio $\nu$. In other words, the elastic-plastic transition first occurs at the dimensionless location $(y / b)_{\mathrm{p}}$ for various Poisson's ratios. Differentiating eqn (9) with respect to the variable $y / b$ and letting the right-hand side equate to zero can get an implicit function of the dimensionless location $\zeta_{0}=(y /$ $b)_{\mathrm{p}}$ of yielding inception versus Poisson's ratio $\nu$, which is expressed as
In order to practice a convenient discussion, eqn (10) could be simplified as a matching parabolic function within the scope of Poisson's ratio by the function approximation technique, which is expressed as

$$
\zeta_{0}=0.2196+2.3349 v-2.4105 v^{2}
$$

The error of the mean square introduced by eqn (11) is less than $0.53 \%$. Fig. 4 presents the fitting result for the values of $\nu$ in the range of 0.2 to 0.5 . Obviously, the approximation function eqn (11) shows a good consistency with eqn (10), in addition, the dimensionless location $\zeta_{0}$ of the elastic-plastic transition on the axis of symmetry performs approximate parabolic growth as $\nu$ increases. Hence, the lower the resistance to the compression of the coating materials, the more shallow the dimensionless location of the yielding inception along the $y$ axis, and the higher the ratio of the yield strength over the maximum contact pressure.

By substituting $y / b$ with $\zeta_{0}$ in eqn (9), then combining eqn (2), (3) and $\left(\sigma_{\mathrm{eq}} / p_{\mathrm{m}}\right)_{\max }=Y / p_{\mathrm{m}}$, the critical load $P_{\mathrm{c}}$ at the elasticplastic transition of the compressed coating is obtained in the form of

$$
P_{\mathrm{c}}=\frac{\pi R Y^{2}\left(1-\nu^{2}\right) C_{v}^{2}}{E}
$$

where $C_{v}$ is given as a function of the Poisson's ratio $\nu$ by

$C_{v}=\sqrt{2}$

$\left(\begin{array}{l}\left(2 \zeta_{0}-\frac{2 \zeta_{0}^{2}}{\left(\zeta_{0}^{2}+1\right)^{1 / 2}}\right)^{2}+\left(2(\nu-1) \zeta_{0}+\frac{1-2 \nu+(2-2 \nu) \zeta_{0}^{2}}{\left(\zeta_{0}^{2}+1\right)^{1 / 2}}\right)^{2} \\ +\left(2 \nu \zeta_{0}+\frac{1-2 \nu-2 \nu \zeta_{0}^{2}}{\left(\zeta_{0}^{2}+1\right)^{1 / 2}}\right)^{(-1 / 2)}\end{array}\right)^{2}$

By substituting eqn (11) into (13), the approximation function of eqn (13) is acquired using a numerical approaching method for the values of $\nu$ in the range of 0.2 to 0.5 , which is written as

$$
\begin{aligned}
& \left(\zeta_{0}-\frac{\zeta_{0}^{2}}{\left(\zeta_{0}^{2}+1\right)^{1 / 2}}\right)\left(2-\frac{4 \zeta_{0}\left(\zeta_{0}^{2}+1\right)^{1 / 2}-2 \zeta_{0}^{3} /\left(\zeta_{0}^{2}+1\right)^{1 / 2}}{\zeta_{0}^{2}+1}\right) \\
& +\left(2(1-\nu) \zeta_{0}+\frac{2(\nu-1) \zeta_{0}^{2}+2 \nu-1}{\left(\zeta_{0}^{2}+1\right)^{1 / 2}}\right)\left(\begin{array}{c}
2(\nu-1) \zeta_{0}\left(\zeta_{0}{ }^{2}+1\right)^{1 / 2} \\
(1-\nu)+\frac{-\left(2(\nu-1) \zeta_{0}^{2}+2 \nu-1\right) \zeta_{0} / 2\left(\zeta_{0}^{2}+1\right)^{1 / 2}}{\zeta_{0}^{2}+1}
\end{array}\right) \\
& +\left(\frac{1-2 \nu \zeta_{0}^{2}-2 v}{\left(\zeta_{0}^{2}+1\right)^{1 / 2}}+2 \nu \zeta_{0}\right)\left(\frac{-2 \nu \zeta_{0}\left(\zeta_{0}^{2}+1\right)^{1 / 2}-\left(1-2 \nu \zeta_{0}^{2}-2 v\right) \zeta_{0} /\left(2\left(\zeta_{0}^{2}+1\right)^{1 / 2}\right)}{\zeta_{0}^{2}+1}+\nu\right)=0
\end{aligned}
$$




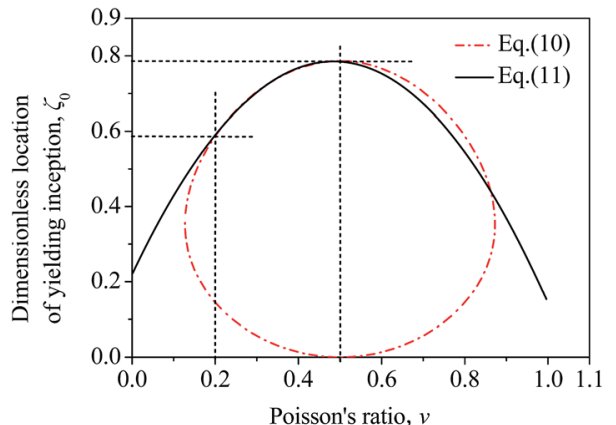

Fig. 4 Parabolic fitting for the dimensionless initial yielding location $v s$. Poisson's ratio.

$$
C_{v}=1.1637+2.9775 \nu-2.9139 v^{2}
$$

The error of the mean square introduced by eqn (14) is less than $0.31 \%$. Replacing $P$ in eqn (3) and (4) with $P_{c}$ in eqn (12) yields the critical half width $b_{\mathrm{c}}$ and the critical interference $\omega_{\mathrm{c}}$ at the yielding inception of the coating material on its axis of symmetry, which are expressed as

$$
b_{\mathrm{c}}=2\left[\frac{P_{\mathrm{c}} R\left(1-\nu^{2}\right)}{\pi E}\right]^{1 / 2}
$$

and

$$
\omega_{\mathrm{c}}=\frac{P_{\mathrm{c}}\left(1-\nu^{2}\right)}{\pi E}\left\{\ln \left[\frac{\pi E}{P_{\mathrm{c}} R\left(1-\nu^{2}\right)}\right]+1\right\}
$$

The critical maximum contact pressure $p_{\mathrm{mc}}$ at the elasticplastic transition can be obtained by substituting eqn (12) and (15) into eqn (2), which is written as

$$
p_{\mathrm{mc}}=C_{v} Y
$$

Eqn (17) illustrates the physical meaning of the dimensionless parameter $C_{v}$, that is, it represents the ratio of the critical maximum contact pressure at the contact centre over the yield strength of the coating material. It is surprising to find that eqn (17) is quite consistent with the conclusions for a spherical contact with a rigid plane, where a Taylor series was adopted to derive the critical contact parameters of the elasticity terminus, and an approximately linear relation between the dimensionless yielding inception depth and Poisson's ratio was obtained for the homogeneous sphere. ${ }^{46}$ Eqn (12), (15) and (16) separately give the critical expressions of load, half width and interference as coating yields along the axis of symmetry. It can be seen that such critical contact parameters are functions of the geometrical and mechanical properties of the loaded cylindrical coating.

\section{Finite element model}

\subsection{Meshing and boundary conditions}

When the coating thickness is thinner, the cylindrical substrate could deform largely under the vertical load of the contact flat, thus having a significant influence on the stress distribution in the coating that should be taken into account. Since the coating and substrate performance give discontinuous mechanical properties at their interface, the research of the coating/ substrate composite should employ a finite element model based on numerical calculation. Fig. 5 presents a plane-strain model of the coated cylinder contacting a rigid flat using the commercial ABAQUS package (version 6.10) on the basis of an implicit integration algorithm, where only a quarter of the strain plane is considered due to axisymmetry. The quarter of a circle represents the cylindrical substrate, and the quarter of a ring represents the shelly coating, in addition the line represents the rigid flat. In order to accurately capture a high stress gradient near the contact zone in a relatively short computation time, the model is divided into four zones (see Fig. 5(a)) by three circular arcs, whose radii from the contact centre are $100 \mu \mathrm{m} /$ $[(R+t) \mu \mathrm{m}], 300 \mu \mathrm{m} /[(R+t) \mu \mathrm{m}]$ and $1500 \mu \mathrm{m} /[(R+t) \mu \mathrm{m}]$, respectively. The first three zones have uniform mesh densities, whose typical sizes are $1 \mu \mathrm{m} /[(R+t) \mu \mathrm{m}], 5 \mu \mathrm{m} /[(R+t) \mu \mathrm{m}]$ and $25 \mu \mathrm{m} /[(R+t) \mu \mathrm{m}]$, respectively. However, the fourth zone displays a gradually coarser mesh with the increasing radius, among which the maximum size adjacent to the base of the substrate is approximately $0.1 R$, and a node is arranged at the bottom of the coating (see Fig. 5(b)). Moreover, the connected partitions have an approximately identical element at their junction so as to increase the convergence of the numerical simulations. A 4-node quadrilateral element (CPE4I) is used for both the coating and substrate. The flat is simulated by an analytical rigid model with no need of defining elements and material properties. The entire mesh consists of 39 020-62 056 elements, and 39 339-62 492 nodes depending on the various $t$ / $R$ ratios of the coating thickness over the substrate radius. The boundary conditions are in agreement with those described in Section 2, that is to say, they contain the node constraints in both the vertical and radial directions at the base of composite system and in the radial direction on the axis of symmetry.

The coating and substrate are assumed to be linear and isotropic materials. The Von Mises yield criterion is used to detect the initiation of plastic deformation. A total of three

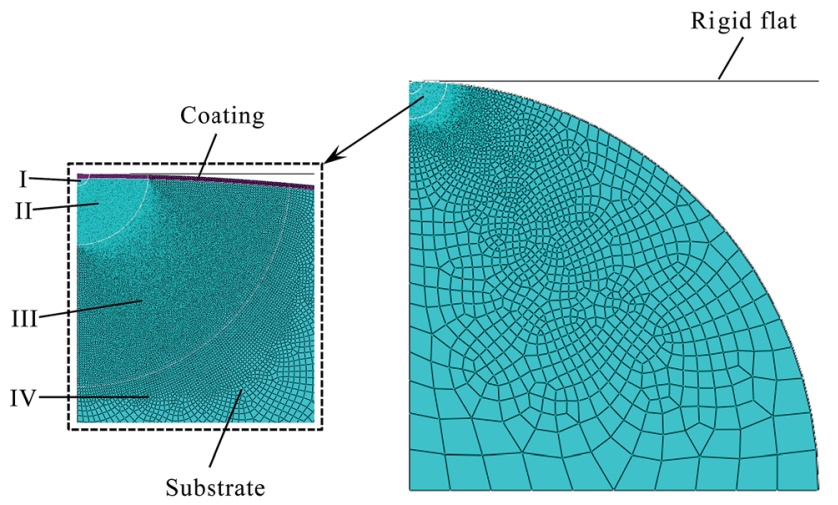

(a)

(b)

Fig. 5 Finite element meshes of the coating/substrate system in (a) partial enlarged view and (b) global view. 
analysis steps are defined throughout the simulation process. One surface-to-surface contact between the rigid flat and coating, which follows a finite sliding formulation, is applied in the initial step, so are the tie constraints imposed between connected zones as well as the coating and substrate. Normal loading is accomplished on the reference point of the rigid flat in the subsequent two load steps. To make the contact relationship begin smoothly, a very small value is applied in the first load step. The increment size and number of iterations in every load step are defined using the default settings of program.

The validity of the numerical model is verified by comparing the finite element results with the analytical Hertz solutions for the elastic contact between an uncoated cylinder and a rigid flat. The error of mean square between the critical loads calculated by Hertz model and finite element method, respectively is less than $0.6 \%$. Proper convergence of the numerical solution is tested by refining the mesh size until further refinement has a negligible effect on the results, increasing individual quantities by at least one order of magnitude. The computation times for each run vary from about $1 \mathrm{~min}$ to $3 \mathrm{~min}$ on a $64 \mathrm{~GB}$ memory HP Z820 workstation with dual hexa-core $2.9 \mathrm{GHz}$ CPU.

\subsection{Results and discussion}

The Von Mises stresses at the onset of the plastic yielding were calculated along the axis of symmetry of the cylindrical coating/ substrate system for various mechanical parameters. Fig. 6 presents such distributions vs. a normalized distance $y / t$ measured from the contact center, where a dashed line denotes the coating/substrate interface at $y / t=1$. The specific simulation parameters are shown in Table 1, in which a third special case of coating material identical to that of the substrate is also presented for comparison. Simulation results show the critical load of the coating $1 /$ substrate system at the yield inception is 7 $\mathrm{kN}$, however the corresponding value of the coating $2 /$ substrate system is about $6.4 \mathrm{kN}$. Furthermore, under the normal load of 7 $\mathrm{kN}$, the Von Mises stresses of both coatings are separately $203 \mathrm{MPa}$ and $274 \mathrm{MPa}$ at the interface, but the corresponding values of both substrates are all $150 \mathrm{MPa}$. Consequently, for the two coated cases, both substrates yield first at the interface, but both coatings are far from their yield strengths. Besides,

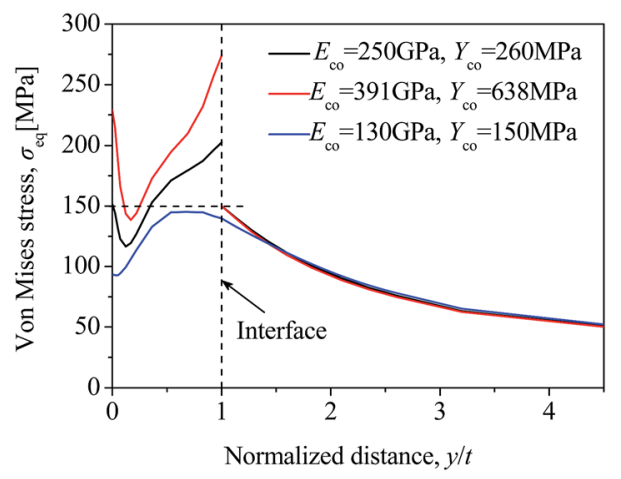

Fig. 6 Equivalent Von Mises stress distribution along the axis of symmetry of a coated cylinder for various coating properties at $t / R=$ 0.001.
Table 1 Simulation parameters of the finite element model

\begin{tabular}{lllll}
\hline & $\begin{array}{l}\text { Elasticity } \\
\text { modulus } \\
E(\mathrm{GPa})\end{array}$ & $\begin{array}{l}\text { Yield strength } \\
Y(\mathrm{MPa})\end{array}$ & $\begin{array}{l}\text { Poisson's } \\
\text { ratio } \nu\end{array}$ & $\begin{array}{l}\text { Geometrical } \\
\text { dimensions }\end{array}$ \\
\hline Substrate & 130 & 150 & 0.32 & $R=10 \mathrm{~mm}$ \\
Coating 1 & 250 & 260 & & $t / R=0.001$ \\
Coating 2 & 391 & 638 & & \\
Coating 3 & 130 & 150 & & \\
\hline
\end{tabular}

a discontinuity in the Von Mises stress distribution is clearly shown at the interface of two cylindrical coating/substrate composites, and the discontinuity is more pronounced for the higher mechanical properties of $E_{\text {co }}=391 \mathrm{GPa}$ and $Y_{\text {co }}=$ $638 \mathrm{MPa}$. For the uniform material case, the normal load is selected to be the same as the upper threshold value for the coating $1 /$ substrate system, i.e., $7 \mathrm{kN}$. Through simulation, the stress distribution along the axis of symmetry is continuous as would be expected, and the maximum stress is only about $145 \mathrm{MPa}$, which is below the yield strength of $150 \mathrm{MPa}$. Therefore, in spite of applying a higher critical load for both coated cylinders, the homogeneous one still undergoes a pure elastic deformation.

Fig. 7 presents the typical locations at the yield inception in the above cylindrical coating/substrate composites under different simulation parameters. For the mechanical properties of $E_{\mathrm{co}}=250 \mathrm{GPa}$ and $Y_{\mathrm{co}}=260 \mathrm{MPa}$, when the geometrical dimension is assigned as $t / R=0.001$, the onset of plastic yielding first occurs within the substrate at the location of $y / t=$ $2.2>1$. Nevertheless, the maximum Von Mises stress in the

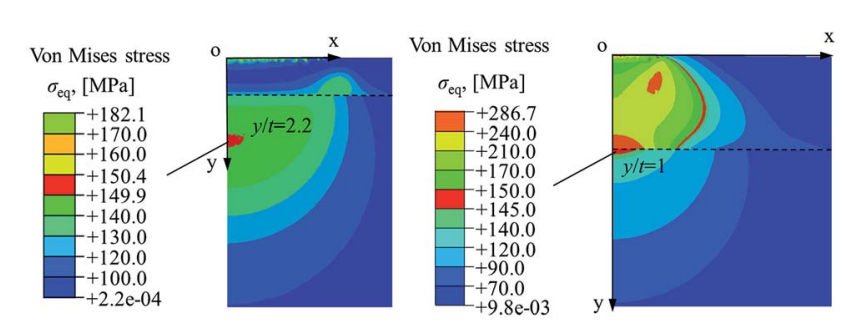

(a)

(b)

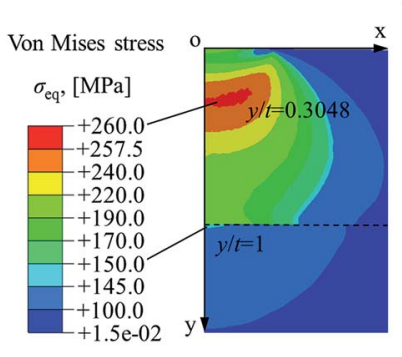

(c)

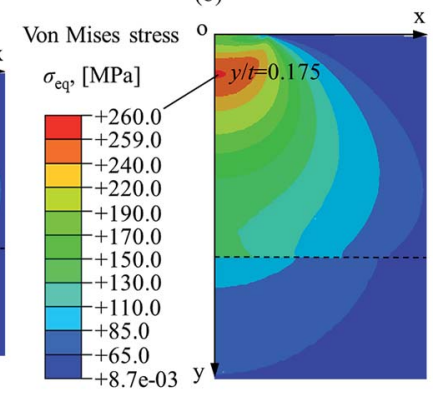

(d)
Fig. 7 Typical locations of yield inception in cylindrical coating/ substrate composites under (a) $E_{\mathrm{co}}=250 \mathrm{GPa}, Y_{\mathrm{co}}=260 \mathrm{MPa}, t / R=$ 0.001 ; (b) $E_{\mathrm{co}}=391 \mathrm{GPa}, Y_{\mathrm{co}}=638 \mathrm{MPa}, t / R=0.005$; (c) $E_{\mathrm{co}}=250$ $\mathrm{GPa}, Y_{\mathrm{co}}=260 \mathrm{MPa}, t / R=0.0105 ;$ and $(\mathrm{d}) E_{\mathrm{co}}=250 \mathrm{GPa}, Y_{\mathrm{co}}=$ $260 \mathrm{MPa}, t / R=0.016$. 
coating is only about $182 \mathrm{MPa}$, being less than the yield strength of $Y_{\text {co }}=260 \mathrm{MPa}$, as shown in Fig. 7(a). As the ratio of the coating thickness over the substrate radius is increased to $t / R=$ 0.0105 , the substrate and coating incipiently produce plastic yielding simultaneously, which occurs at the interface for the substrate, while that arises at $y / t=0.3048$ for the coating (see Fig. $7(\mathrm{c})$ ). However, at the ratio of $t / R=0.016$, the coating first yields to plastic deformation at $y / t=0.175<1$, but the maximum Von Mises stress of the substrate is far below its yield strength $Y_{\mathrm{su}}=150 \mathrm{MPa}$ (see Fig. 7(d)). In addition, for the coating properties of $E_{\mathrm{co}}=391 \mathrm{GPa}, Y_{\mathrm{co}}=638 \mathrm{MPa}$ and $t / R=$ 0.005 , as the substrate generates an initial yielding at the interface, the coating is under a pure elastic deformation in all regions (see Fig. 7(b)).

According to the above analysis, the homogeneous cylinder manifests a superior yielding resistance in comparison with both the coated ones. Besides, for the coating properties of $E_{\text {co }}=448 \mathrm{GPa}, Y_{\text {co }}=836 \mathrm{MPa}$ and $t / R=0.003$, the substrate initially yields at the interface on the axis of symmetry, and the critical yielding load is about $6.4 \mathrm{kN}$. However, when the coating material acquires the property of substrate, the corresponding critical load and yield position are $8.02 \mathrm{kN}$ and $y / t=0.9$ respectively. Obviously, the dimensionless coordinate of the initial yield point moves from $y / t<1$ to the interface with the substrate as the top properties of the homogeneous cylinder are replaced by the coating material, and the admissible load, resulting in a pure elastic deformation, becomes lower for the coated cylinder. Accordingly, it is observed that the coating weakens the yielding resistance of a cylindrical composite from the cases in the presence and absence of it. Actually, this degeneracy is mainly attributed to the additional stresses for relatively thin coatings induced by binding constraints at the coating/substrate interface, which could lower the critical load of a coated system and shift the first yielding location closer to the interface, in contrast to that without them.

For the purpose of deeply understanding the action mechanism of the bond conditions imposed at the coating/substrate interface, Fig. 8 presents the principal stresses of both heterogeneous and homogeneous cylinders along their axis of symmetry for the properties described, i.e., $E_{\mathrm{co}}=448 \mathrm{GPa}, Y_{\mathrm{co}}=$ $836 \mathrm{MPa}$ and $t / R=0.003$ with the substrate nature unchanged. The normal loads applied in the two analyses are identical and equal to the critical value of the coated cylinder. As is known, the shear stress components are $\tau_{x y}=\tau_{x z}=\tau_{y z}=0$ for the axis of symmetry of the cylinder, and hence the corresponding normal stress components $\sigma_{x}, \sigma_{y}$ and $\sigma_{z}$ are three principal stresses. For the heterogeneous cylinder (Fig. 8(a)), the distributions of $\sigma_{x}$ and $\sigma_{z}$ show a clear discontinuity at $y / t=1$. Moreover, the principal stress $\sigma_{x}$ of the coating possesses a value of $\sigma_{x}=$ $156 \mathrm{MPa}$, which is tensile, while that of substrate equals to $-6.3 \mathrm{MPa}$ becoming compressive. In the axial direction, the principal stress $\sigma_{z}$ remains compressive at both sides of the interface, but its value jumps from $-7.8 \mathrm{MPa}$ to $-58 \mathrm{MPa}$ as $y / t$ increases. Along the axis of symmetry, the principal stress $\sigma_{y}$ is continuous and compressive with a value of $-175 \mathrm{MPa}$ at the interface. In the second case (Fig. 8(b)), all principal stresses are compressive and decrease monotonically to zero as $y / t$ comes

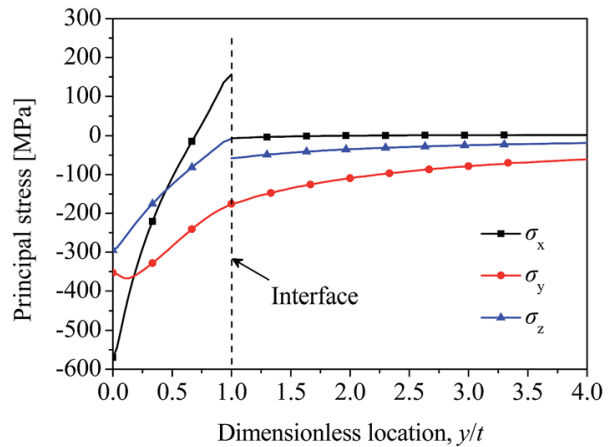

(a)

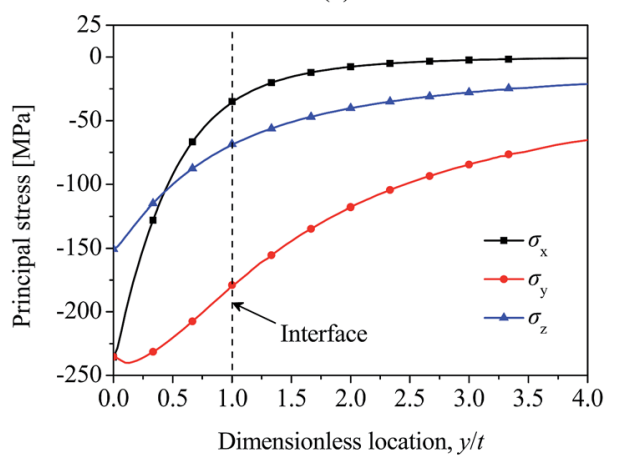

(b)

Fig. 8 Principal stresses along the axis of symmetry of cylinders with coating properties of (a) $E_{\mathrm{co}}=448 \mathrm{GPa}, Y_{\mathrm{co}}=836 \mathrm{MPa}$ and (b) $E_{\mathrm{co}}=$ $130 \mathrm{GPa}, Y_{\mathrm{co}}=150 \mathrm{MPa}$ under the substrate parameters of $E_{\mathrm{su}}=130$ $\mathrm{GPa}, Y_{\mathrm{su}}=150 \mathrm{MPa}$ at $t / R=0.003$.

close to the higher end. At the interface, $\sigma_{x}, \sigma_{y}$ and $\sigma_{z}$ are continuous and their values are $-35 \mathrm{MPa},-179 \mathrm{MPa}$, and -68 MPa respectively. It can be seen that for the principal stress $\sigma_{y}$, there is a negligible distinction at $y / t=1$ for both cases, however, the principal stresses $\sigma_{x}$ and $\sigma_{z}$ of the coated cylinder are substantially different from those without the coating. Through a comparative study on the two systems in the presence and absence of the coating, the bonding conditions force both the coating and substrate to keep an equal radial displacement at the interface. However, it is difficult for the stiffer coating to follow an inward constriction of the more compliant substrate. Thus, the coating is stretched by the substrate and then reduces the compressive stress of the substrate from $-35 \mathrm{MPa}$ to $-6.3 \mathrm{MPa}$ at their interface. In the axial direction, both the coating and substrate maintain no deformation for the plane-strain model, so the principal stress $\sigma_{z}=\nu\left(\sigma_{x}+\sigma_{y}\right)$ according to Hooke's law. It is not hard to find that decreases about $\nu$ times less than $\sigma_{x}$ does. It is known that the Von Mises stress is proportional to the quadratic sum of $\sigma_{x}$ $-\sigma_{y}, \sigma_{z}-\sigma_{y}, \sigma_{x}-\sigma_{z}$.

Nevertheless, as the discontinuity is produced at the interface, the difference of $\sigma_{x}-\sigma_{y}$ increases from $144 \mathrm{MPa}$ to 168.7 $\mathrm{MPa}$, and $\sigma_{z}-\sigma_{y}$ increases from 110.5 MPa to $117 \mathrm{MPa}$ in the substrate. Since $\sigma_{x}-\sigma_{z}=\sigma_{x}-\nu \sigma_{x}-\nu \sigma_{y}$, so the increment of $\sigma_{x}-\sigma_{z}$ is about $(1-\nu)$ times to that of $\sigma_{x}$, namely, it increases from $33 \mathrm{MPa}$ to $51.7 \mathrm{MPa}$. Hence, the Von Mises stress increases from $130 \mathrm{MPa}$ to $150 \mathrm{MPa}$, with these differences of principal 
stresses increasing due to the bonding conditions at the substrate/coating interface, resulting in an additional increase of $20 \mathrm{MPa}$, as mentioned before. Furthermore, increasing $E_{\text {co }}$ can enhance the interaction between the coating and the substrate at the interface, thus bringing about a higher level of additional stresses. As a result, the yielding resistance of the coated cylinder becomes even lower and the discontinuity at the interface is more pronounced. This phenomenon may explain some experimental observations of the delamination failure for relatively thin coatings. The stress discontinuity at the interface and the weakening effect of the coating/substrate system could also be observed in a coated sphere contacting a rigid flat, ${ }^{47,48}$ but the coated sphere expresses an equal stress distribution in the $x$-axis and $z$-axis directions at any $y$ coordinate, i.e., $\sigma_{x}=\sigma_{z}$, which is different from the stress relation $\sigma_{z}=\nu\left(\sigma_{x}+\sigma_{y}\right)$ for the coated cylinder. Not only that, the coated sphere has the same stress-strain principle in planes containing $y$-axis due to the symmetry, but the coated cylinder performs the properties only in the cross-section.

Under loading conditions, the total interference of the cylindrical coating/substrate composite consists of the individual interferences of the coating and substrate. Fig. 9 presents the dimensionless critical interference $\omega_{\text {c_comb }} / \omega_{c}$ of the composite at yielding inception and the corresponding components $\omega_{\mathrm{c} \_ \text {co }} / \omega_{\mathrm{c}}$ and $\omega_{\mathrm{c} \_ \text {su }} / \omega_{\mathrm{c}} v s$. the ratio of the coating thickness over the substrate radius, where $\omega_{\mathrm{c}}$ denotes the critical interference of a homogeneous cylinder with the substrate properties identical to those of the coating for a relative study. For the coating properties of $E_{\text {co }}=250 \mathrm{GPa}, Y_{\text {co }}=260 \mathrm{MPa}$, $\omega_{\mathrm{c} \_ \text {su }} / \omega_{\mathrm{c}}$ is much larger than $\omega_{\mathrm{c} \_ \text {co }} / \omega_{\mathrm{c}}$ when $t / R$ takes a small value, besides, $\omega_{\text {c_comb }} / \omega_{\mathrm{c}}$ and $\omega_{\mathrm{c} \_ \text {su }} / \omega_{\mathrm{c}}$ increase first and then decrease but are always greater than 1 around $t / R=0.01 . \omega_{\mathrm{c} \_c o} /$ $\omega_{\mathrm{c}}$, however, expresses monotonous growth from 0 with $t / R$ increasing, which indicates the total interference of the coated cylinder is mainly attributed to the substrate deformation under a thinner coating. As $t / R$ increases, $\omega_{\mathrm{c}_{-} s u} / \omega_{\mathrm{c}}$ decreases continuously and $\omega_{\text {c_co }} / \omega_{\mathrm{c}}$ keeps rising, while at the ratio of $t / R=0.15$, they have an identical value. However, when the $t / R$ value is near the higher end of the scale, the relative value $\omega_{c_{-} \_o} / \omega_{c}$ of the

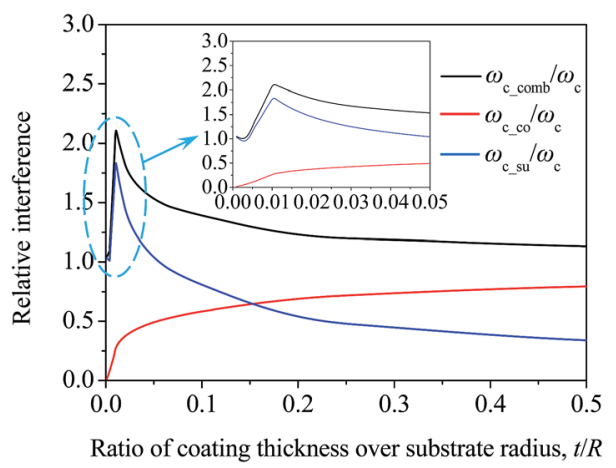

Fig. 9 Critical interference of the coated cylinder relative to the value of a homogeneous one at the yielding inception and the corresponding components with the increasing ratio of coating thickness over substrate radius.

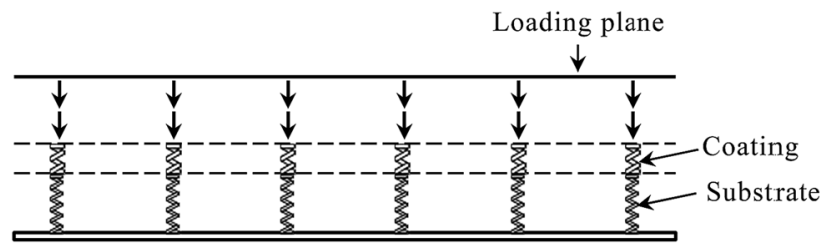

Fig. 10 Spring series model of the cylindrical coating/substrate system under a normal contact load.

coating approaches 1 and the relative value $\omega_{\mathrm{c}_{-} \text {su }} / \omega_{\mathrm{c}}$ of the substrate closes to 0 , which reveals that the coating displacement displays as a dominant contributor to the system interference, while the contribution of the substrate is almost negligible at large $t / R$ values. Therefore, the total interference $\omega_{\text {c_comb }}$ of the coated cylinder is close to the critical value of the homogeneous cylinder when $t / R$ is specified as a great value, that is, $\omega_{\mathrm{c} \_ \text {comb }} / \omega_{\mathrm{c}}$ approaches 1 with increasing $t / R$.

Analogously, the cylindrical coating/substrate composite could be perceived as being composed of two springs in series in the load direction, both of which experience the overall load $P$, as shown in Fig. 10. When $t / R$ is very small, the stiffness of the coating is much larger than that of the substrate, thus the system interference takes place primarily in the substrate. With the value of $t / R$ becoming greater, the substrate stiffness becomes comparable to the coating stiffness, and the coating interference increases with the substrate contraction decreasing. When $t / R$ has a large value, the coating stiffness is almost negligible in comparison to that of the substrate, so the coating compression serves as the dominant contributor to system interference.

\section{Conclusions}

The yielding inception of a coated cylinder contacting a rigid plane was studied by Hertz theory and finite element solutions. Some conclusions can be drawn as follows:

(1) For super-thick coatings, the critical expressions of load, half width and interference were derived at the onset of plastic yielding along the axis of symmetry by a function approximation method. It can be seen that the geometrical and mechanical properties of the loaded cylindrical coating have important impacts on these critical contact parameters. Moreover, the smaller the Poisson's ratio of the coating, the more shallow the dimensionless location of the yielding inception along the $y$ axis, and the higher the ratio of the yield strength over the maximum contact pressure.

(2) For coatings with a thinner thickness, the Von Mises stress is discontinuous at the coating/substrate interface, and the discontinuity is more pronounced as the distinction of coating and substrate in their mechanical properties is increased. The initial yield position may be located either within the coating, in the substrate or at the interface, with the ratio of coating thickness over substrate radius changing.

(3) An upper elasticity modulus of the coating in comparison with that of the substrate can bring about an additional stress at 
the interface, thus lowering the yielding resistance of the coated cylinder.

(4) When $t / R$ is very small, the total interference of the coated cylinder is mainly attributed to the substrate deformation. With the $t / R$ ratio becomes greater, the coating compression becomes comparable to the substrate value. As $t / R$ has larger values, the coating contraction serves as a dominant contributor to the composite interference.

(5) The coated cylinder expresses some similar behaviors as those of a coated sphere loaded by a rigid plane, but it gets the characteristics only in the cross-section, while the coated sphere possesses the corresponding properties in all symmetry planes containing the load direction.

\section{Acknowledgements}

The authors gratefully acknowledge the financial support from the National Natural Science Foundation of China (Grant no. 51202261 \& 51475449), National Basic Research Program of China (973 Program) (Grant no. 2013CB632302) and State Key Laboratory for Mechanical Behavior of Materials Foundation (Grant no. 20141605).

\section{References}

1 M. Cui, J. Pu, J. Liang, L. Wang, G. Zhang and Q. Xue, RSC Adv., 2015, 5, 104829-104840.

2 D. He, S. Zheng, J. Pu, G. Zhang and L. Hu, Tribol. Int., 2015, 82, 20-27.

3 D.-W. Kim and K.-W. Kim, Wear, 2014, 315, 95-102.

4 Q. Li, Z. Feng, L. Liu, J. Sun, Y. Qu, F. Li and M. An, RSC Adv., 2015, 5, 12025-12033.

5 A. F. Rousseau, J. G. Partridge, E. L. H. Mayes, J. T. Toton, M. Kracica, D. G. McCulloch and E. D. Doyle, Surf. Coat. Technol., 2015, 272, 403-408.

6 K. R. Sriraman, P. Manimunda, R. R. Chromik and S. Yue, RSC Adv., 2016, 6, 17360-17372.

7 Y. Ye, Y. Wang, H. Chen, J. Li, Y. Yao and C. Wang, Tribol. Int., 2015, 90, 362-371.

8 S. Zhou, H. Chen and L. Ma, Surf. Coat. Technol., 2014, 242, 177-182.

9 J. A. Brandao, J. H. O. Seabra and M. J. D. Castro, Measurement, 2016, 91, 479-487.

10 T. H. Machado and K. L. Cavalca, Appl. Math. Model., 2016, 40, 9546-9564.

11 G. M. Roach and L. L. Howell, J. Mech. Des., 2002, 124, 485491.

12 T. Liu, A. Jagota and C.-Y. Hui, Soft Matter, 2015, 11, 38443851.

13 S. Saito, T. Ochiai, F. Yoshizawa and M. Dao, Sci. Rep., 2016, 6, 34063 .

14 F. Jin and X. Guo, Int. J. Solids Struct., 2010, 47, 1508-1521.

15 O. I. Zhupanska and A. F. Ulitko, J. Mech. Phys. Solids, 2005, 53, 975-999.

16 H. Qiu, D. A. Hills, D. Nowell and D. Dini, Int. J. Mech. Sci., 2008, 50, 293-298.
17 D. Li, Y.-L. Han, Y.-M. Hu and R.-H. Wang, Power Eng., 2016, 175-180.

18 C. Pereira, A. Ramalho and J. Ambrosio, Multibody Syst. Dyn., 2015, 35, 277-298.

19 Y. P. Chiu and M. J. Hartnett, J. Tribol., 1987, 109, 479-485.

20 S. C. Hunter, J. Appl. Mech., 1961, 28, 611-617.

21 T. Doca, F. M. Andrade Pires and J. M. A. Cesar de Sa, Int. J. Solids Struct., 2014, 51, 1697-1715.

22 T. Doca and F. M. Andrade Pires, Tribol. Int., 2014, 79, 92-98.

23 L. Chen and S. H. Chen, J. Appl. Phys., 2014, 116, 164701.

24 P. Meijers, Appl. Sci. Res., 1968, 18, 353-383.

25 S. Stupkiewicz, J. Lengiewicz, P. Sadowski and S. Kucharski, Tribol. Int., 2016, 93, 511-522.

26 H. Proudhon, J. Savkova, S. Basseville, V. Guipont, M. Jeandin and G. Cailletaud, Wear, 2014, 311, 159-166.

27 T. Hong and N. Saka, Wear, 1991, 148, 47-68.

28 H. Tian and N. Saka, Wear, 1991, 148, 261-285.

29 P. K. Gupta and J. A. Walowit, J. Lubr. Technol., 1974, 96, 250257.

30 Y. Alinia, M. A. Guler and S. Adibnazari, Mech. Res. Commun., 2014, 55, 45-52.

31 M. A. Guler and F. Erdogan, Int. J. Mech. Sci., 2007, 49, 161182.

32 Y. Alinia, M. A. Guler and S. Adibnazari, Mech. Mater., 2014, 68, 207-216.

33 M. A. Guler, Y. Alinia and S. Adibnazari, Mech. Mater., 2013, 66, 134-159.

34 S. J. Cole and R. S. Sayles, J. Tribol., 1992, 114, 334-340.

35 K. Komvopoulos, J. Tribol., 1989, 111, 430-439.

36 J. Yang and K. Komvopoulos, J. Tribol., 2004, 126, 18-27.

37 Z. Q. Gong and K. Komvopoulos, J. Tribol., 2004, 126, 655663.

38 R. K. Njiwa, R. Consiglio and J. von Stebut, Surf. Coat. Technol., 1998, 102, 138-147.

39 H. H. Bennani and J. Takadoum, Surf. Coat. Technol., 1999, 111, 80-85.

40 H. Djabella and R. D. Arnell, Thin Solid Films, 1993, 223, 8797.

41 R. Jahedi and S. Adibnazari, Int. J. Adv. Des. Manuf. Technol., 2015, 8, 1-12.

42 N. Demirhan and B. Kanber, Mech. Base. Des. Struct. Mach., 2013, 41, 383-398.

43 M. A. Guler, Y. Alinia and S. Adibnazari, Int. J. Mech. Sci., 2012, 64, 62-81.

44 K. L. Johnson, Contact Mechanics, Cambridge University Press, Cambridge, 1985, pp. 96-119.

45 C. M. Pereira, A. L. Ramalho and J. A. Ambrósio, Nonlinear Dyn., 2011, 63, 681-697.

46 V. Brizmer, Y. Kligerman and I. Etsion, Int. J. Solids Struct., 2006, 43, 5736-5749.

47 R. Goltsberg and I. Etsion, Wear, 2013, 308, 10-16.

48 R. Goltsberg, I. Etsion and G. Davidi, Wear, 2011, 271, 29682977. 